\title{
Naringin promotes differentiation of bone marrow stem cells into osteoblasts by upregulating the expression levels of microRNA-20a and downregulating the expression levels of PPAR $\gamma$
}

\author{
JIFENG FAN, JIE LI and QINBO FAN \\ Department of Orthopedics, The First Affiliated Hospital of Dalian Medical University, \\ Dalian, Liaoning 116011, P.R. China
}

Received September 26, 2014; Accepted May 28, 2015

DOI: $10.3892 / \mathrm{mmr} .2015 .3996$

\begin{abstract}
Naringin is a dihydrotestosterone flavonoid compound that significantly inhibits bone loss, improves bone density, and enhances biomechanical anti-compression performance. Previous studies have demonstrated that naringin improves the activity levels of osteocalcin (OC) and alkaline phosphatase (ALP) in MC3T3-E1 osteoblast precursor cells. The present study investigated the effects of naringin on osteoblastic differentiation and inhibition of adipocyte formation in bone marrow stem cells (BMSCs). The levels of osteogenesis were modulated via upregulation of the expression levels of microRNA (miR)-20a, and downregulation of the expression levels of peroxisome proliferator-activated receptor $\gamma(\operatorname{PPAR} \gamma)$. The results indicated that naringin significantly enhanced BMSC proliferation in a dose-dependent manner. In addition, naringin significantly increased the mRNA expression levels of OC, ALP, and collagen type I. Furthermore, naringin decreased the protein expression levels of PPAR $\gamma$, and increased the expression levels of miR-20a in the BMSCs. These results suggested that miR-20a may regulate the expression of PPAR $\gamma$ in BMSCs. To our knowledge, this is the first study to report naringin-induced osteogenesis via upregulation of the expression levels of miR-20a, and downregulation of the expression levels of PPAR $\gamma$. These results indicated the important role of naringin in BMSC differentiation.
\end{abstract}

\section{Introduction}

Bone marrow stem cells (BMSCs) are currently under active investigation due to their therapeutic potential for bone tissue engineering and cell replacement therapy (1). BMSCs

Correspondence to: Mr. Qinbo Fan, Department of Orthopedics, The First Affiliated Hospital of Dalian Medical University, 222 Zhong Shan Road, Dalian, Liaoning 116011, P.R. China

E-mail: qinbofanhj@163.com

Key words: naringin, bone marrow stem cells, peroxisome proliferator-activated receptor $\gamma$, microRNA-20a were initially described in 1966, when they were isolated and cultured from bone marrow rat cartilage cells (2). A previous study showed that BMSCs migrate toward damaged bone tissue, thereby demonstrating the therapeutic potential of BMSCs for tissue injury repair and cell replacement therapy (3). In addition, BMSCs have been reported to survive in high hypoxic/ischemic inflammatory environments (4). The present study aimed to investigate the mechanism underlying BMSC migration, as well as the role of BMSCs in tissue repair-associated protein regulation. This was achieved by modulating BMSC motility and viability through genetic engineering $(5,6)$.

Peroxisome proliferator activated receptor $\gamma(\operatorname{PPAR} \gamma)$ is a subtype of PPAR, which mediates BMSC differentiation into adipocytes (7). PPAR $\gamma$ is a ligand-activated nuclear transcription factor, which is involved in cellular differentiation, growth, and apoptosis (8). PPAR $\gamma$ mediates the differentiation of BMSCs into adipocytes, and an increase in the protein expression levels of PPAR $\gamma$ results in a significant increase in adipocyte differentiation, concomitant with reduced levels of osteogenic differentiation, in the bone marrow (9). Therefore, PPAR $\gamma$ may prove useful in the treatment and prevention of osteoporosis, a disease in which deregulated BMSC differentiation, dedifferentiation, and transdifferentiation leads to a decrease in the number of osteoblasts, and an increase in the number of adipocytes (10).

Micro (mi)RNAs are small (19-22 nucleotides) endogenous non-coding RNA molecules, which suppress the expression of target genes (11). miRNAs have an important role in numerous biological processes, including cellular proliferation, differentiation, and apoptosis. Previous studies have shown that the upregulation of miR-20a expression leads to an increase in the protein expression levels of the adipogenic PPAR $\gamma$ marker gene in rats, as compared with the controls $(12,13)$.

Naringin is a dihydrotestosterone flavonoid compound, which markedly inhibits bone loss, improves bone density, and enhances biomechanical anti-compression performance (14). Naringin has been shown to promote the proliferation and differentiation of MC3T3-E1 osteoblast precursor cells in vitro; however, it does not promote bone mineralization (15). High concentrations of naringin increase the synthesis and activity levels of alkaline phosphatase (ALP), promote osteoblast 
differentiation, and stimulate bone formation (16). A previous study demonstrated that naringin increases the activity levels of osteocalcin (OC) and ALP, and promotes the proliferation and differentiation of MC3T3-E1 osteoblast precursor cells, without affecting bone mineralization (16).

The present study aimed to investigate whether naringin was able to enhance BMSC differentiation and inhibit adipocyte formation by modulating osteogenesis via the upregulation of miR-20a expression, and the downregulation of PPAR $\gamma$ expression.

\section{Materials and methods}

Drugs and reagents. Naringin (Fig. 1) at a purity $>95 \%$ (Sigma-Aldrich, St Louis, MO, USA) was dissolved in physiological saline, according to the manufacturer's instructions. Dulbecco's modified Eagle's medium (DMEM) and fetal bovine serum (FBS) were purchased from Gibco Life Technologies (Carlsbad, CA, USA), and Invitrogen Life Technologies (Carlsbad, CA, USA), respectively. MTT assay and Lipofectamine ${ }^{\circledR} 2000$ were purchased from Sigma-Aldrich.

Animals and BMSC isolation. Male and female New Zealand white rabbits (4-8 weeks old) weighing $2.0 \pm 0.5 \mathrm{~kg}$ were provided by the Experimental Animal Center of Dalian Medical University (Dalian, China). The rabbits were maintained in individual cages at $21-23^{\circ} \mathrm{C}$, under a $12 \mathrm{~h}$ light-dark cycle (8:00; 20:00) and humidity level of 55-65\%. The study was approved by the ethics committee of The First Affiliated Hospital of Dalian Medical University (Dalian, China). The rabbits were anesthetized by intravenous injection of $30 \mathrm{mg} / \mathrm{kg}$ pentobarbital (Invitrogen Life Technologies). A total of 2-3 ml bone marrow was subsequently collected from the lateral tibial tubercle of the rabbits using a puncture needle. The bone marrow cell suspension was then mixed with an equal volume of Percoll solution (Duke Scientific, Palo Alto, CA, USA), forming a clear interface. Density gradient centrifugation was performed at 1,200 $\mathrm{x} g$ for $20 \mathrm{~min}$, in order to isolate the BMSCs. The supernatant was discarded and the mononuclear BMSCs were extracted. The BMSCs were washed with phosphate-buffered saline (PBS) and further centrifuged at $1,000 \mathrm{x}$ g for $10 \mathrm{~min}$. The supernatant was discarded and $10 \mathrm{ml}$ DMEM supplemented with 15-20\% FBS was added to the precipitate, prior to incubation at $37^{\circ} \mathrm{C}$ in a humidified atmosphere containing $5 \% \mathrm{CO}_{2}$. The cells were cultured until they reached $80 \%$ confluence and were then used for subsequent experimentation.

Cell proliferation assay. The levels of BMSC proliferation were determined using an MTT assay. BMSCs were seeded at a density of $2.0 \times 10^{4}$ cells/well into 96 -well plates at $37^{\circ} \mathrm{C}$ in a humidified atmosphere containing $5 \% \mathrm{CO}_{2}$. The BMSCs were then treated with either $0.01,0.1,1,10$ and $100 \mu \mathrm{M}$ naringin (17) for $48 \mathrm{~h}$, or with medium containing $0,0.1,1$, and $10 \mu \mathrm{M}$ naringin for 3, 7, 14 and 21 days. For the MTT assay, $10 \mu \mathrm{l}$ MTT was added to each well prior to incubation at $37^{\circ} \mathrm{C}$ in a humidified atmosphere containing $5 \% \mathrm{CO}_{2}$ for $4 \mathrm{~h}$. The medium was discarded, and $150 \mu \mathrm{l}$ dimethyl sulfoxide (Beyotime Institute of Biotechnology, Nanjing, China) was added to the solution prior to further incubation at room temperature with gentle agitation for $10 \mathrm{~min}$. The optical density of the solution was read at $570 \mathrm{~nm}$ using a microplate reader (Sunrise, Tecan Trading AG, Männedorf, Switzerland).

Quantification of the protein expression levels of PPAR by western blot analysis. BMSCs were treated with various concentrations of naringin $(0.1,1$, and $10 \mu \mathrm{M})$ for $48 \mathrm{~h}$. The protein expression levels of PPAR $\gamma$ were then determined by western blotting. The protein concentrations were determined using a Novagen ${ }^{\circledR}$ Bicinchoninic Acid Protein Assay kit (Novagen, Merck Millipore, Darmstadt, Germany). Protein was extracted from cells using Cell Lysis Buffer (Cell Signaling Technology, Inc., Danvers, MA, USA). Equal quantities of protein was separated by $10 \%$ SDS-polyacrylamide gel and blotted onto polyvinylidene fluoride membranes (Millipore). The primary antibodies used for the western blot analysis were as follows: Anti-PPAR $\gamma$ (cat. no. 2435S; 1:500; Cell Signaling Technology, Inc.) and anti- $\beta$-actin (cat. no. sc-69879; 1:2,000; Santa Cruz Biotechnology, Inc., Dallas, TX, USA) at $4^{\circ} \mathrm{C}$ overnight. Subsequently, membranes were washed and incubated with a horseradish peroxidase-conjugated anti-rabbit IgG secondary antibody (cat. no. C520011; 1:20,000, Sangon Biotech Co., Ltd., Shanghai, China). The blots were visualized using an enhanced chemiluminescence assay kit (GE Healthcare Life Sciences, Little Chalfont, UK), and analyzed using VersaDoc Gel Imaging system (Bio-Rad Laboratories, Inc., Hercules, CA, USA).

Quantification of the mRNA expression levels of $O C, A L P$, collagen type I (Col I), and miR-20a by reverse transcription-quantitative polymerase chain reaction. In order to determine the mRNA expression levels of OC, ALP, and $\mathrm{Col} \mathrm{I}$, the BMSCs were treated with $1 \mu \mathrm{M}$ naringin for $3,7,14$ and 21 days or with medium containing $0,0.1,1$, and $10 \mu \mathrm{M}$ naringin for $48 \mathrm{~h}$. Total RNA was extracted from the BMSCs using TRIzol ${ }^{\circledR}$ reagent (Invitrogen Life Technologies). A total of $1 \mu \mathrm{g}$ total RNA was subsequently reverse transcribed into cDNA using an RTq-PCR mixture (Takara Biotechnology Co., Ltd., Dalian, China). A 5 ng cDNA template was used to carry out the reaction. Various primers (Sangon Biotech Co., Ltd.) were used in order to amplify OC, ALP, and Col I (Table I). In order to determine the expression levels of miR-20a, the BMSCs were treated with $0.1,1$, and $10 \mu \mathrm{M}$ naringin for $48 \mathrm{~h}$. Total RNA containing miR-20a was extracted from the BMSCs using a miRNeasy RNA Isolation kit (Bogoo Biomart, Shanghai, China). The expression levels of miR-20a were subsequently analyzed by RT-qPCR qPCR (7500 Real-Time PCR system; Applied Biosystems, Foster City, CA, USA) according to the manufacturer's instructions. PCR cycling conditions were as follows: 35 cycles at $95^{\circ} \mathrm{C}$ for $30 \mathrm{sec}, 60^{\circ} \mathrm{C}$ for $45 \mathrm{sec}$ and $72^{\circ} \mathrm{C}$ for $50 \mathrm{sec}$. The sequence of the miR-20a primer is shown in Table I. cDNA was reverse transcribed from 100 ng total miRNA using a QuantiTect Reverse Transcription kit (Bogoo Biomart). mRNA expression levels were determined using the $2^{-\Delta \Delta C t}$ method of relative quantification.

BMSC transfection with miR-20a and anti-miR-20a. miR-20a precursor and anti-miR-20a (Ambion Life Technologies, Carlsbad, CA, USA) were synthesized by Sangon Biotech Co., Ltd. miR-20 a precursor: 5'-GTAGTAGCACTAAAGTGC TTATAGTGCAAGTAGTGTTTAGTTATCTACTGCATTAT 
Table I. Primer design for reverse transcription-quantitative polymerase chain reaction.

Gene

Primers

Annealing temperature (cycle)

\begin{tabular}{ll}
\hline Osteocalcin & F: 5'-CATGAGAGCCCTCACA-3' \\
& R: 5'-AGAGCGACACCCTAGAC-3' \\
Alkaline phosphatase & F: 5'-TCAGAAGCTCAACACCAACG-3' \\
& R: 5'-GTCAGGGACCTGGGCATT-3' \\
Collagen type I & F: 5'-TGACCTCAAGATGTGCCACT-3' \\
& R: 5'-GGGAGTTTCCATGAAGCCAC-3' \\
microRNA-20a & F:5'-AAGAATTTAAATAAAAAAAAAGAACA-3' \\
& R: 5'-CACGGGCTGAGGAAAATA-3' \\
$\beta$-actin & F:5'-GCTCTCCAGAACATCACTCCTGCC-3' \\
& R:5'-CGTTGTCATACCAGGAAATGAGCTT-3'
\end{tabular}

F, foward; R, reverse.

G AGC ACT TA A AGT ACT GC-3'; and 3'-GCA GTACTTTAAGTGCTCATAATGCAGTAGATAACTAAAC ACTACCTGCACTATAAGCACTTTAGTGCTA-5'; anti - miR-20a, 5'-CTA A AC ACT ACC T GC ACT ATA AGCACTTTAGTGCTAC-3', and 5'-GAAATGTACTGCGCG TGGAGACGTTTTGGCCACTGAC-3'. The BMSCs were

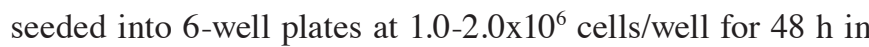
order to perform the transfection. The cells were transfected using Lipofectamine ${ }^{\circledR} 2000$ with 100 ng miR-20a precursor, anti-miR-20a, or a negative precursor (pcDNA3.1+). After $24 \mathrm{~h}$, the BMSCs were treated with $0.1,1$, and $10 \mu \mathrm{M}$ naringin for $48 \mathrm{~h}$. In all experiments untreated BMSCs were used as controls.

Statistical analysis. All statistical analyses were performed using SPSS 17.0 (SPSS, Inc., Chicago, IL, USA). The data are presented as the mean \pm standard deviation. A $\chi^{2}$ and exact probability test were performed in order to compare two groups. $\mathrm{P}<0.05$ was considered to indicate a statistically significant difference.

\section{Results}

Effects of naringin on the proliferation of BMSCs. As shown in Fig. 2, naringin dose-dependently enhanced the proliferation of BMSCs. Following treatment with $0.1,1,10$ and $100 \mu \mathrm{M}$ naringin for $48 \mathrm{~h}$, BMSC proliferation was significantly enhanced (Fig. 2A). In addition, treatment with 0.1 , 1, and $10 \mu \mathrm{M}$ naringin for 3-10 days significantly enhanced BMSC proliferation (Fig. 2B).

mRNA expression levels of OC, ALP, and Col I in BMSCs. BMSCs were treated with $1 \mu \mathrm{M}$ naringin for 3, 7, 14 and 21 days. There was a significant increase in the mRNA expression levels of OC, ALP, and Col I in the naringin-treated BMSCs, as compared with the control group (Fig. 3A-3C). Following treatment with $1 \mu \mathrm{M}$ naringin for 21 days, the mRNA expression levels of $\mathrm{OC}$ were the highest, with expression levels 5.8-fold greater, as compared with that of the control cells (Fig. 3A). The mRNA expression levels of ALP were increased by 2.3, 2.4,

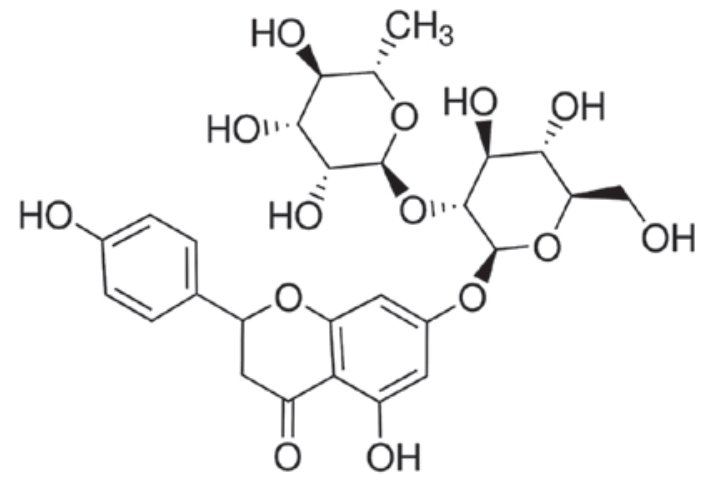

Figure 1. Chemical structure of naringin.

3.9 and 2.9-fold following 3, 7, 14, and 21 days, respectively, as compared with the control group (Fig. 3B). The mRNA expression levels of Col I significantly increased between 14-21 days during osteogenic differentiation, as compared with the control group (Fig. 3C). In addition, BMSC treatment with 0.1, 1, and $10 \mu \mathrm{M}$ naringin for $48 \mathrm{~h}$ significantly increased the mRNA expression levels of OC, ALP, and Col I (Fig. 3D).

Protein expression levels of PPAR $\gamma$ in BMSCs. Following treatment with $0,0.1,1$, and $10 \mu \mathrm{M}$ naringin for $48 \mathrm{~h}$, the protein expression levels of PPAR $\gamma$ were analyzed by western blotting (Fig. 4A). Treatment with 1 and $10 \mu \mathrm{M}$ naringin significantly decreased the protein expression levels of PPAR $\gamma$, as compared with the control group (Fig. 4B).

RT-qPCR analysis of miR-20a expression in BMSCs. Following treatment with $0,0.1,1$, and $10 \mu \mathrm{M}$ naringin for $48 \mathrm{~h}$, the expression levels of miR-20a in the BMSCs were analyzed by RT-qPCR. The expression levels of miR-20a were significantly increased in the BMSCs treated with naringin (1 and $10 \mu \mathrm{M}$ ), as compared with the control group (Fig. 5).

Overexpression of $m i R-20$ a suppresses the expression levels of PPAR $\gamma$ in BMSCs. In order to determine whether miR-20a regulates the protein expression of PPAR $\gamma$ in BMSCs, the 


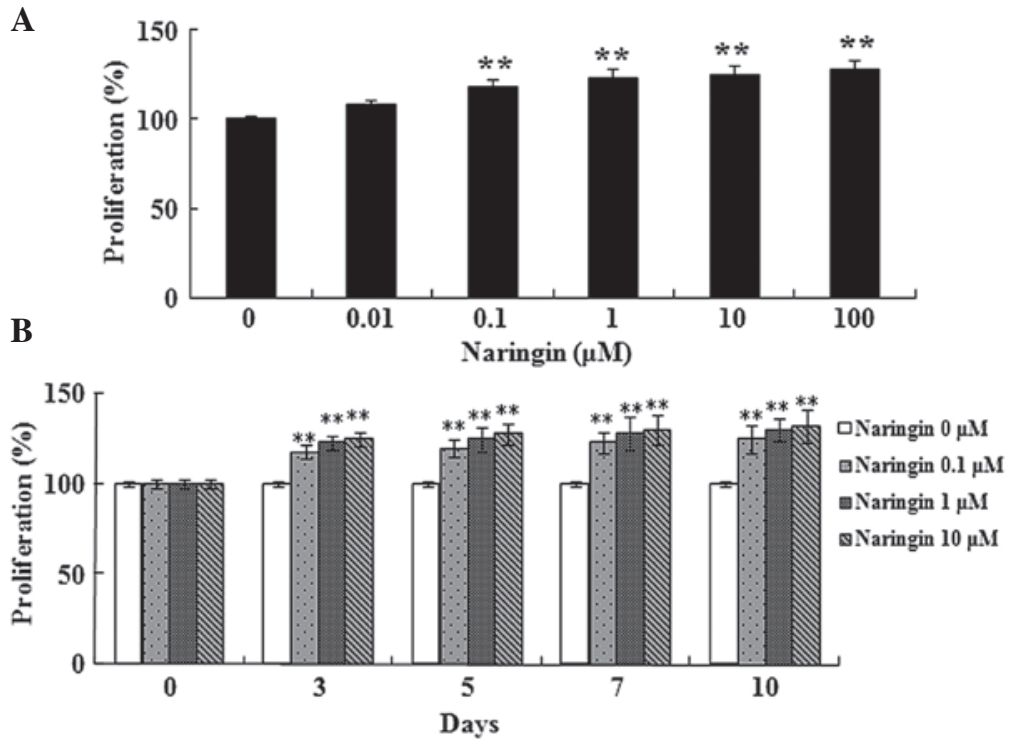

Figure 2. Effects of naringin on the proliferation of bone marrow stem cells (BMSCs). (A) Treatment with naringin enhanced the proliferation of BMSCs after $48 \mathrm{~h}$ in a dose-dependent manner. (B) Naringin concentrations ranging from 0.1-10 $\mu \mathrm{M}$ stimulated BMSC proliferation after 3-10 days. ${ }^{* *} \mathrm{P}<0.01$, vs. the control group.
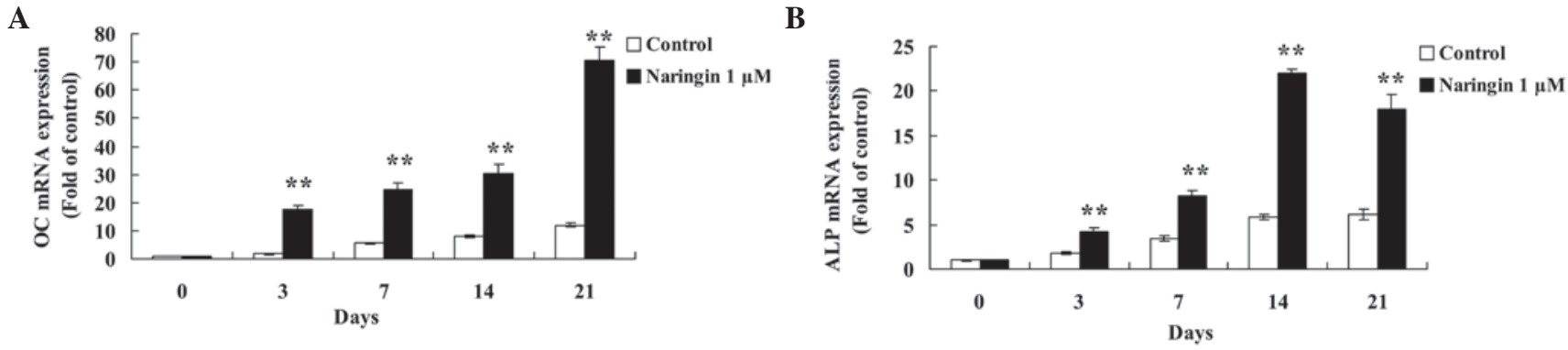

$\mathbf{C}$

D
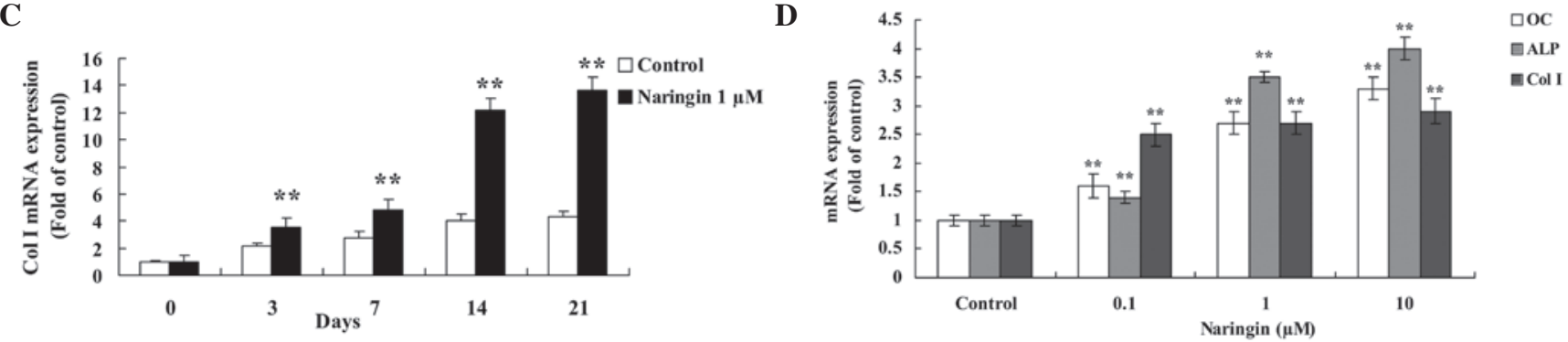

Figure 3. mRNA expression levels of osteocalcin (OC), alkaline phosphatase (ALP) and collagen type I (Col I) in bone marrow stem cells (BMSCs). The mRNA expression levels of (A) OC, (B) ALP, and (C) Col I in the BMSCs following treatment with $1 \mu \mathrm{M}$ naringin for 21 days. (D) Treatment with $0.1,1$, and $10 \mu \mathrm{M}$ naringin for $48 \mathrm{~h}$ significantly increased the mRNA expression levels of OC, ALP, and Col I. ${ }^{* *} \mathrm{P}<0.01$, vs. the control group.

A

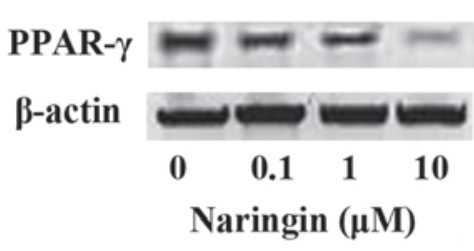

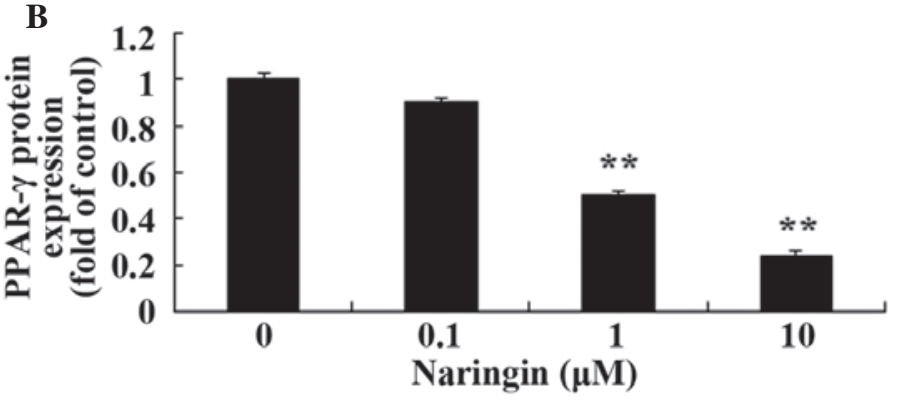

Figure 4. Protein expression levels of peroxisome proliferator-activated receptor $\gamma(\operatorname{PPAR} \gamma$ ). (A) Following treatment with $0,0.1,1$, and $10 \mu \mathrm{M}$ naringin for $48 \mathrm{~h}$, the protein expression levels of PPAR $\gamma$ in the bone marrow stem cells (BMSC) were analyzed using western blot analysis. (B) Protein expression levels of PPAR $\gamma$ as determined by statistical analysis. ${ }^{\text {** }} \mathrm{P}<0.01$, vs. the control group. 


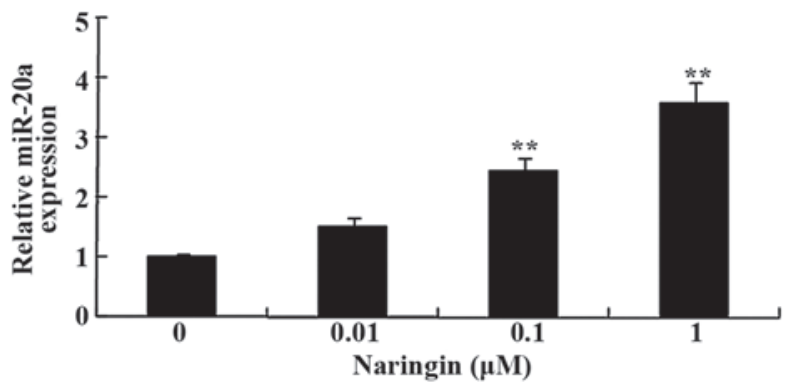

Figure 5. Reverse transcription-quantitative polymerase chain reaction (RT-qPCR) analysis of microRNA (miR)-20a expression levels. Following treatment with $0.1,1$, and $10 \mu \mathrm{M}$ naringin for $48 \mathrm{~h}$, the miR-20a expression levels of the bone marrow stem cells were analyzed by RT-qPCR. ${ }^{*} \mathrm{P}<0.01$, vs. the control group.

A

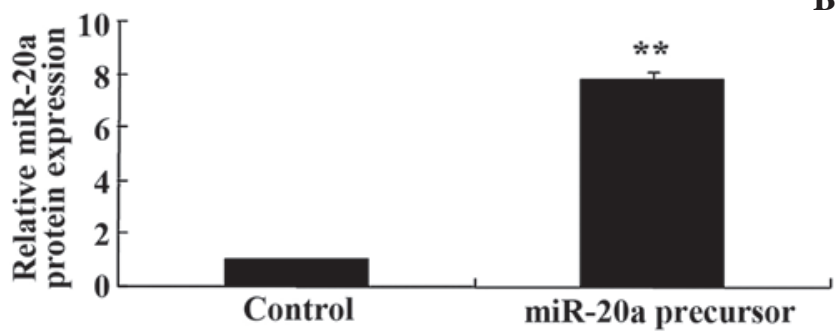

B

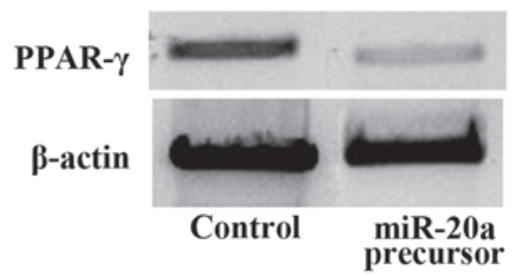

Figure 6. Overexpression of microRNA (miR)-20a suppressed the protein expression levels of proliferation agent activated receptor $\gamma$ (PPAR $\gamma$ ). (A) The effects of the microRNA (miR)-20a precursor on the expression levels of miR-20a expression were determined by RT-qPCR. (B) miR-20a decreased the protein expression levels of peroxisome proliferator-activated receptor $\gamma(\operatorname{PPAR} \gamma)$, as determined by western blotting." $\mathrm{P}<0.01$, vs. the control group.

A

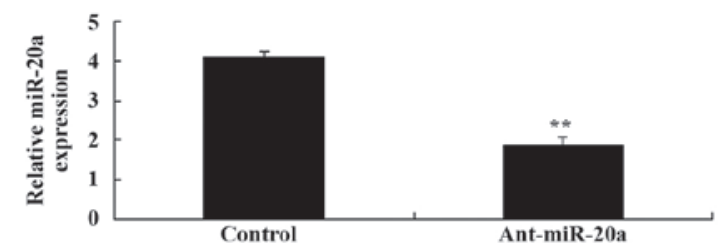

C

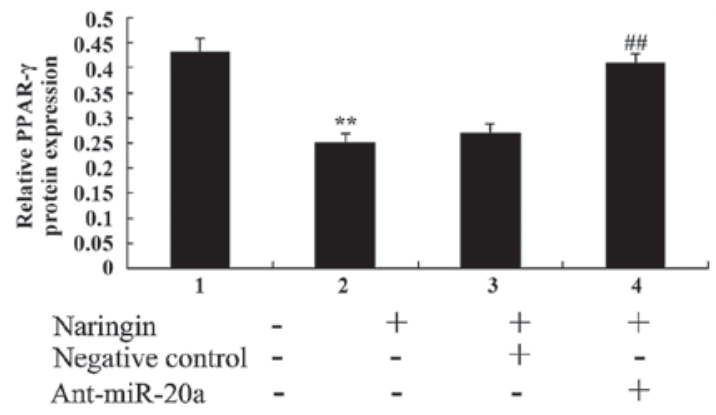

B

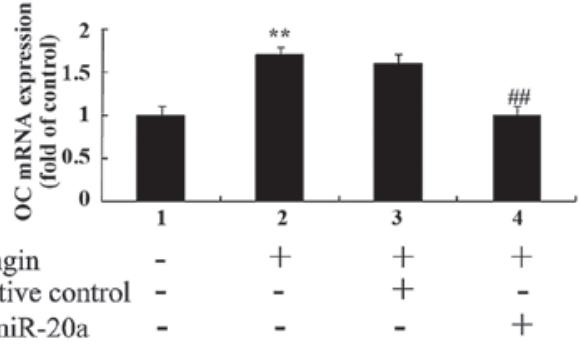

D

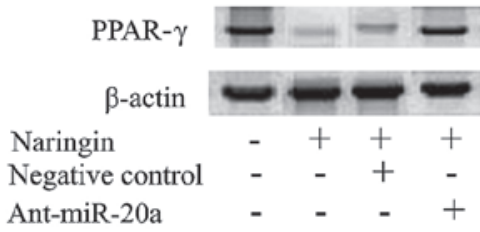

Figure 7. The reversal effects of anti-microRNA (miR)-20a on naringin. (A) Transfection of the bone marrow stem cells (BMSCs) with anti-miR-20a significantly reduced the expression levels of miR-20a. (B) Transfection with anti-miR-20a suppressed the effects of $1 \mu \mathrm{M}$ naringin on the mRNA expression levels of osteocalcin (OC) in BMSCs. (C) Protein expression levels of peroxisome proliferator-activated receptor $\gamma$ (PPAR $\gamma$ ). (D) Transfection with anti-miR-20a increased the protein expression levels of PPAR $\gamma$ at $48 \mathrm{~h}$. ${ }^{* *} \mathrm{P}<0.01$, vs. the control group, and ${ }^{\# \#} \mathrm{P}<0.01$, vs. the naringin-treated group transfected with negative control.

protein expression levels of PPAR $\gamma$ were analyzed in BMSCs post-transfection with a miR-20a precursor. The effects of the miR-20a precursor on the expression levels of miR-20a expression were analyzed by RT-qPCR. BMSC transfection with a miR-20a precursor significantly increased the expression levels of miR-20a in BMSCs (Fig. 6A). Conversely, the protein expression levels of PPAR $\gamma$ were significantly decreased following transfection with miR-20a (Fig. 6B).
Anti-miR-20a reverses the effects of naringin in BMSCs. Anti-miR-20a antibody was used to investigate the effects of miR-20a on the naringin-induced inhibition of BMSCs. BMSC transfection with anti-miR-20a antibody significantly reduced the expression levels of miR-20a in BMSCs (Fig. 7A). In addition, BMSC transfection with anti-miR-20a antibody also significantly reduced the effects of $1 \mu \mathrm{M}$ naringin on the mRNA expression levels of OC in BMSCs (Fig. 7B), and 
significantly increased the protein expression levels of PPAR $\gamma$ (Fig. 7C) at $48 \mathrm{~h}$. These results suggest that the anti-miR20a may neutralize the inhibitory effects of naringin through the downregulation of miR-20a expression, and the upregulation of PPAR $\gamma$ expression in BMSCs (Fig. 7D).

\section{Discussion}

BMSCs are adult stem cells located in the bone marrow, which have high self-renewal and differentiation potentials (18). Although BMSCs have been isolated and cultured from numerous tissue types, such as the liver, embryonic blood, umbilical cord blood, and amniotic fluid, BMSCs are predominantly obtained from bone marrow tissues $(19,20)$. In the bone marrow, BMSCs account for $0.001 \%-0.1 \%$ of the total cells, and exhibit high differentiation potential. Indeed, BMSCs are able to differentiate into adipocytes, bone cells, cartilage cells, and myoblasts (21). BMSCs have been isolated from the bone marrow in numerous species, including mice, rats, rabbits, and humans. The results of the present study demonstrated that naringin was able to dose-dependently enhance the proliferation of BMSCs, which prompted the further investigation of the therapeutic effects of naringin on BMSCs.

Osteoblasts determine not only the rate of bone formation, they also adjust the activity levels of osteoclasts, thereby determining the rate of bone resorption (22). Bone formation includes three stages: Osteoblast proliferation, osteoblast maturation, and mineralization of the extracellular matrix. Osteoblast proliferation is the bone formation step that predominantly determines the final amount of formed bone. ALP is an enzyme secreted during the differentiation of osteoblasts (16). The levels of ALP are correlated with the synthesis of Col I and with the formation of bone matrix; ALP therefore serves as a marker for the early and mid-stages of osteoblast differentiation (23). In addition, the activity levels of ALP are correlated with the activity levels of osteoblasts (24). Previous studies demonstrated that mid to high concentrations of naringin are able to increase the synthesis and activity levels of ALP, thereby demonstrating that ALP promotes osteoblast differentiation and bone formation $(25,26)$. Naringin has previously been shown to significantly increase the amount of bone morphogenetic protein- 2 in bone cells, and promote osteoblast differentiation into osteogenic cells (27). The results of the present study demonstrated that naringin is able to significantly increase the mRNA expression levels of OC, ALP, and Col I. The expression levels of OC following treatment with $1 \mu \mathrm{M}$ naringin for 21 days were the highest. In addition, treatment with $1 \mu \mathrm{M}$ naringin significantly increased the expression levels of ALP after 3 days. Furthermore, the mRNA expression levels of Col I significantly increased following treatment with $1 \mu \mathrm{M}$ naringin between 14-21 days.

PPAR $\gamma$ is the primary regulator of adipocyte differentiation, which has an important role in the regulation of BMSC differentiation (28). A recent study demonstrated that PPAR $\gamma$ has an important role in the gene therapy of osteoporosis (29). The results of the present study determined that treatment with 1 and $10 \mu \mathrm{M}$ naringin for $48 \mathrm{~h}$ decreased the protein expression levels of PPAR $\gamma$ in the BMSCs. Furthermore, treatment with 1 and $10 \mu \mathrm{M}$ naringin for $48 \mathrm{~h}$ significantly increased the expression levels of miR-20a in the BMSCs. The present study also investigated whether miR-20a regulated the protein expression levels of PPAR $\gamma$ following transfection of BMSCs with a miR-20a precursor or anti-miR-20a. The results indicated that miR-20a regulated the protein expression levels of PPAR $\gamma$ in BMSCs.

In conclusion, the results of the present study revealed that naringin is able to promote BMSC differentiation into osteoblasts, via the upregulation of miR-20a, and the downregulation of PPAR $\gamma$. Thus suggesting that naringin may be a potential novel drug that may promote BMSC differentiation into osteoblasts, in the treatment of osteoporosis.

\section{References}

1. Wu G, Cui Y, Ma L, Pan X, Wang X and Zhang B: Repairing cartilage defects with bone marrow mesenchymal stem cells induced by CDMP and TGF- $\beta 1$. Cell Tissue Bank 15: 51-57, 2014.

2. Gong P, Wang Y, Zhang J and Wang Z: Bone marrow mesenchymal stem cells in hepatocellular carcinoma. Front Biosci (Landmark Ed) 18: 811-819, 2013.

3. Sotiropoulou PA, Perez SA, Salagianni M, Baxevanis CN and Papamichail M: Characterization of the optimal culture conditions for clinical scale production of human mesenchymal stem cells. Stem Cells 24: 462-471, 2006.

4. Hu X, Wei L, Taylor TM, et al: Hypoxic preconditioning enhances bone marrow mesenchymal stem cell migration via Kv2.1 channel and FAK activation. Am J Physiol Cell Physiol 301: C362-C372, 2011.

5. Pelled G, G T, Aslan H, Gazit Z and Gazit D: Mesenchymal stem cells for bone gene therapy and tissue engineering. Curr Pharm Des 8: 1917-1928, 2002.

6. Damoulis PD, Drakos DE, Gagari E and Kaplan DL: Osteogenic differentiation of human mesenchymal bone marrow cells in silk scaffolds is regulated by nitric oxide. Ann NY Acad Sci 1117: 367-376, 2007 .

7. Floyd ZE and Stephens JM: Controlling a master switch of adipocyte development and insulin sensitivity: Covalent modifications of PPAR $\gamma$. Biochim Biophys Acta 1822: 1090-1095, 2012.

8. Krishnan A, Nair SA and Pillai MR: Biology of PPAR gamma in cancer: A critical review on existing lacunae. Curr Mol Med 7: 532-540, 2007.

9. Takada I, Suzawa M, Matsumoto K and Kato S: Suppression of PPAR transactivation switches cell fate of bone marrow stem cells from adipocytes into osteoblasts. Ann NY Acad Sci 1116: 182-195, 2007.

10. Takada I and Kato S: Molecular mechanism of switching adipocyte / osteoblast differentiation through regulation of PPAR-gamma function. Clin Calcium 18: 656-661, 2008 (In Japanese).

11. Salmanidis M, Pillman K, Goodall G and Bracken C: Direct transcriptional regulation by nuclear microRNAs. Int J Biochem Cell Biol 54: 304-311, 2014.

12. Yu F, Cui Y, Zhou X, Zhang X and Han J: Osteogenic differentiation of human ligament fibroblasts induced by conditioned medium of osteoclast-like cells. Biosci Trends 5: 46-51, 2011.

13. Zhang JF, Fu WM, He ML, Xie WD, Lv Q, Wan G, Li G, Wang H, Lu G, Hu X, et al: MiRNA-20a promotes osteogenic differentiation of human mesenchymal stem cells by co-regulating BMP signaling. RNA Biol 8: 829-838, 2011.

14. Li N, Jiang Y, Wooley $\mathrm{PH}, \mathrm{Xu} \mathrm{Z}$ and Yang SY: Naringin promotes osteoblast differentiation and effectively reverses ovariectomy-associated osteoporosis. J Orthop Sci 18: 478-485, 2013.

15. Chen LL, Lei LH, Ding PH, Tang Q and Wu YM: Osteogenic effect of Drynariae rhizoma extracts and Naringin on MC3T3-E1 cells and an induced rat alveolar bone resorption model. Arch Oral Biol 56: 1655-1662, 2011.

16. Surampalli G, K Nanjwade B and Patil PA: Corroboration of naringin effects on the intestinal absorption and pharmacokinetic behavior of candesartan cilexetil solid dispersions using in-situ rat models. Drug Dev Ind Pharm: June 11, 2014 (Epub ahead of print).

17. Kim HJ, Song JY, Park HJ, Park HK, Yun DH and Chung JH: Naringin protects against rotenone-induced apoptosis in human neuroblastoma SH-SY5Y Cells. Korean J Physiol Pharmacol 13: 281-285, 2009. 
18. Heo JS, Choi SM, Kim HO, Kim EH, You J, Park T, Kim E and Kim HS: Neural transdifferentiation of human bone marrow mesenchymal stem cells on hydrophobic polymer-modified surface and therapeutic effects in an animal model of ischemic stroke. Neuroscience 238: 305-318, 2013.

19. Ge Z, Goh JC and Lee EH: The effects of bone marrow-derived mesenchymal stem cells and fascia wrap application to anterior cruciate ligament tissue engineering. Cell Transplant 14: 763-773, 2005.

20. Lee OK, Kuo TK, Chen WM, Lee KD, Hsieh SL and Chen TH: Isolation of multipotent mesenchymal stem cells from umbilical cord blood. Blood 103: 1669-1675, 2004.

21. Mukhopadhyay A: Perspective on liver regeneration by bone marrow-derived stem cells-a scientific realization or a paradox. Cytotherapy 15: 881-892, 2013.

22. Shao J, Zhang Y, Yang T, Qi J, Zhang L and Deng L: HIF-1alpha disturbs osteoblasts and osteoclasts coupling in bone remodeling by up-regulating OPG expression. In Vitro Cell Dev Biol Anim: April 10, 2015 (Epub ahead of print).

23. Aubin JE: Advances in the osteoblast lineage. Biochem Cell Biol 76: 899-910, 1998.

24. Suzuki E, Ochiai-Shino H, Aoki $\mathrm{H}$, et al: Akt activation is required for TGF-beta1-induced osteoblast differentiation of MC3T3-E1 pre-osteoblasts. PLoS One 9: e112566, 2014.
25. Kumar VS, Rajmane AR, Adil M, Kandhare AD, Ghosh P and Bodhankar SL: Naringin ameliorates acetic acid induced colitis through modulation of endogenous oxido-nitrosative balance and DNA damage in rats. J Biomed Res 28: 132-145, 2014.

26. Li L, Zeng Z and Cai G: Comparison of neoeriocitrin and naringin on proliferation and osteogenic differentiation in MC3T3-E1. Phytomedicine 18: 985-989, 2011.

27. Wu JB, Fong YC, Tsai HY, Chen YF, Tsuzuki M and Tang $\mathrm{CH}$ : Naringin-induced bone morphogenetic protein-2 expression via PI3K, Akt, c-Fos/c-Jun and AP-1 pathway in osteoblasts. Eur J Pharmacol 588: 333-341, 2008.

28. Sun J, Wang Y, Li Y and Zhao G: Downregulation of PPAR $\gamma$ by miR-548d-5p suppresses the adipogenic differentiation of human bone marrow mesenchymal stem cells and enhances their osteogenic potential. J Transl Med 12: 168, 2014

29. Harsløf T, Tofteng CL, Husted LB, Nyegaard M, Børglum A, Carstens M, Stenkjær L, Brixen K, Eiken P, Jensen JE, et al: Polymorphisms of the peroxisome proliferator-activated receptor gamma (PPAR $\gamma$ ) gene are associated with osteoporosis. Osteoporos Int 22: 2655-2666, 2011. 